\title{
LAS CONSTRUCCIONES PASIVAS DE NOMINATIVO CON INFINITIVO EN LATÍN CLÁSICO. ESTUDIO SINTÁCTICO
}

The purpose of this paper is to study the devices which can explain the existence of the passive construction Nominatiuus cum Infinitiuo (NcI) in Latin. The evidence of the data makes it possible to assume that NcI construction is the regular passive transformation of double nominal complements when the main verb is impressive, while a predicative complement structure is in the origin of $\mathrm{NcI}$ when a declarative verb is concerned. It is suggested as conclusion that this kind of passive obeys to the same transformative devices as all passive structures.

0. Para el estudio del funcionamiento de las construcciones pasivas de Nominativo con Infinitivo ( NcI) voy a partir de la suposición, tradicionalmente admitida ${ }^{1}$, de que la voz pasiva es una transformación de la activa. He de advertir, no obstante, que este supuesto es meramente metodológico, no explicativo. Con ello quiero decir que utilizo el término «transformación» no para explicar los mecanismos mediante los cuales generan los hablantes las frases en cuestión, sino como denominación de una relación regular y sistematizable entre dos estructuras de la lengua. En este sentido es obvio que la pasiva es una transformación de la activa en cuanto que para toda frase pasiva se puede encontrar su correspondiente activa en función de determinados mecanismos sintácticos.

1. La voz pasiva de los verbos personales latinos presenta ciertas peculiaridades que apuntan hacia una interpretación especial de este fenómeno lingüístico o, al menos, diferente de la que precisa el mismo en las lenguas románicas. Para empezar hay que tener en cuenta que la pasivización en latín afecta no sólo a los verbos tradicionalmente llamados transitivos, sino también a un gran número de los que en activa o no presentan

1 Cf. por ejemplo C. García Gual, El sistema diatético del verbo griego, Madrid 1970 , p. 42. 
complemento o lo presentan marcado con casos distintos del acusativo. La gramaticalidad de frases como

(1) Caes. B.G. III 21, 1 pugnatum est diu atque acriter.

(2) Cic. Cat. III 37 mihi nihil ab istis noceri potest.

(3) Cic. Verr. IV 96 uia, qua Assaro itur Hennam.

añadidas a las que podrían llamarse pasivas regulares, es decir, relacionadas con activas del tipo

(4) puer patrem amat.

ha hecho pensar a algunos estudiosos de este tema que en latin la pasiva denota, ante todo, un proceso de intransitivación. Así Rubio ${ }^{2}$ identifica los verbos pasivos con verbos de estado, basándose, sobre todo, en la morfología de los paradigmas analíticos; la cópula est es estática por excelencia.

2. El elemento que determina la forma que ha de adoptar una frase pasiva es la marca casual que presenta el complemento de la activa; un complemento en acusativo de la voz activa se convierte en sujeto en nominativo de la pasiva, dando lugar a pasivas con sujeto; un complemento en otro caso permanece invariable, produciendo una pasiva sin sujeto o pasiva impersonal. La construcción impersonal es la que corresponde también a las frases que en activa se complementan con una oración completiva, cualquiera que sea la forma de ésta. El resto de los cambios que se operan en la frase activa y que afectan, como es bien sabido, al sujeto y al verbo, son los mismos para todas las frases. En realidad la presencia o ausencia de sujeto en la pasiva, dato relacionado con la forma del complemento en la activa, es el único criterio que permite una clasificación interna de las pasivas en latín ${ }^{3}$.

3. Sin embargo, existe un tipo de distribución pasiva cuya explicación por medio de este principio general de transformación, presenta dificultades concretas. Se trata de la construcción de Nominativo con Infinitivo $(\mathrm{NcI})$, del tipo

(5) Cic. C.M. 63 consurrexisse omnes illi dicuntur.

Las dificultades de interpretación están motivadas por los puntos siguientes:

2 Introducción a la Sintaxis estructural del latin, I, Barcelona 1966, p. 93.

3 M. Bassols, Sintaxis Latina, I, Madrid 1976, p. 272 ss. 
1) Estas construcciones proceden de frases activas de verbo regente con complementación de Acusativo con Infinitivo (AcI), analizado tradicionalmente como oración completiva. La frase (5) procedería de

(5a) consurrexisse omnes illos dicit.

El hecho de que esta construcción activa, que, si es una oración, debe constituir una unidad estructural, pueda "desmembrarse» en sus dos componentes primarios - Acusativo, por un lado, e Infinitivo, por otro- en la transformación pasiva, plantea un grave problema para la interpretación sintáctica de las estructuras de AcI en activa.

2) La construcción pasiva de NcI es para algunos verbos la única posible; en cambio para otros, alterna con una construcción impersonal de verbo regente pasivo $+\mathrm{Acl}$ en condiciones dificiles de precisar.

3) En general, se ha considerado que en los verbos que admiten la coexistencia de construcciones personales e impersonales ya no es gramatical la estructura activa con complemento acusativo (*dico eos), por lo que la construcción de $\mathrm{NcI}$ ha de explicarse, aparentemente, mediante criterios específicos y diferentes de los del resto de la pasiva.

Estos tres son los problemas básicos a los que habría que encontrar solución para lograr una comprensión cabal de las construcciones de $\mathrm{NcI}$.

4. Los verbos que documentan esta forma de pasiva son de dos clases:

a) Verbos impresivos: iubeo, ueto, sino, cogo, prohibeo 4 .

b) Verbos de lengua, declarativos, de pensamiento y de percepción: dico, trado, credo, uideo etc.

4.1. Los primeros adoptan la construcción de $\mathrm{NcI}$ con exclusividad:

(6) Cic. Sest. 95 accusare eum ... non situs est.

(7) Liu. XXX 34,8 cum Carthaginienses ... cogerentur manus conserere.

(8) Liu. X 32,4 ubi et intrare ipsi Samnitium agrum prohiberentur.

(9) Liu. VII 36,11 iussae legiones arma capere.

(10) Cic. Quir. 13 equites Romani flere uetabantur.

No aparecen atestiguadas construcciones impersonales de verbo regente pasivo + AcI (*iubetur eos ire). La complementación activa de estos verbos se ha considerado tradicionalmente como oracional de AcI, a pesar de datos como (6)-(10). Sin embargo es un hecho que frases pasivas

4 Estos son los verbos que citan Ernout-Thomas (Syntaxe Latine, París 1953, p. 330) para ilustrar este tipo de estructura, aunque no son los únicos. Impero y otros verbos de orden presentan el mismo comportamiento. Cf. M.E. Torrego, El sistema de la oración completiva latina. AcI en Tito Livio, tesis doctoral inédita, Madrid 1984, p. 172 ss. 
como éstas sugieren un replanteamiento del análisis de sus correspondientes activas. El hecho de que en pasiva el acusativo no permanezca como sujeto sintáctico del infinitivo, sino que pase a nominativo sujeto de su verbo regente, tiene una sola interpretación sintáctica: tampoco en activa el acusativo es sujeto del infinitivo, sino que ha de analizarse como complemento del verbo principal; esto explica que tome parte en la transformación pasiva, pasando a sujeto del verbo personal. El infinitivo realizaría la función de segundo complemento en una estructura de complementación doble de la voz activa. Acusativo e infinitivo no constituirian, pues, una estructura oracional, porque no mantienen entre sí relaciones de Sujeto-Predicado ${ }^{5}$, sino que estarían regidos individualmente por el verbo regente.

4.2. Admitir ese análisis para estos verbos no presenta una excesiva dificultad, porque pueden considerarse como verbos de dos argumentos ${ }^{6}$, en tanto en cuanto el acusativo sea de persona y el infinitivo activo, de modo que pueda establecerse entre ellos una relación semántica de agenteacción. En estas condiciones, esta clase verbal rige complementos de infinitivo incluso en lenguas donde el infinitivo no constituye nunca el predicado de una oración completiva. El castellano, por ejemplo, presenta estructuras paralelas a las latinas:

(11) Liu. XXVIII 40,14 uincere ego prohibui Hannibalem

(11a) "yo he impedido a Aníbal vencer».

Según los criterios establecidos por A.M. Bolkestein ${ }^{7}$ para la caracterización semántica del complemento de un verbo de orden, a saber, que sea de persona, que sea el agente de la acción que se ordena y que tenga control sobre ella, estas frases pueden ser analizadas como de complementación doble, acusativo de persona + nombre de acción, porque el acusativo aparece dotado de los rasgos semánticos que precisa para ser el complemento de un verbo de orden. Ahora bien, ¿es posible mantener el mismo análisis cuando el acusativo no cumple tales requisitos semánticos? Los casos donde esto ocurre son de dos tipos:

5 Otro indicio para esta afirmación es la invariabilidad que presenta el infinitivo en estas distribuciones, que parece responder a una indiferencia en la expresión del modo que no se produce en otras distribuciones, y que identifica al infinitivo con el nomen actionis más que con un predicado verbal. Cf. M.E. Torrego, El sistema..., loc. cit.

- De hecho, las estructuras de Acusativo + Infinitivo son conmutables con Dativo $+u t+$ Subj.

7 «The relation between Form and Meaning of latin subordinate Clauses governed by verba dicendi», Mnemosyne 29, 1976, p. 279 ss. 
a) el acusativo no tiene control sobre el infinitivo porque éste aparece en voz pasiva:

(12) Liu. VI 15,6 in uincla te duci iubebo.

b) el acusativo no es de persona:

(13) Liu. XXXIV 2,4, si coetus et concilia et secretas consultationes esse sinas.

Este tipo de estructuras analizadas como de doble complementación son imposibles en castellano:

(12a) * «te ordenaré ser llevado a prisión»

(13a) * «si permites existir a las consultas secretas».

En estos casos se opta tradicionalmente por un análisis oracional de las frases activas, según el cual el acusativo sería el sujeto del infinitivo, habida cuenta de que no puede ser, por sus rasgos semánticos, complemento del verbo de orden. Ahora bien, las consecuencias de este análisis, si fuera correcto, deberían hacerse sentir en las transformaciones pasivas; este tipo de frases debería adoptar en voz pasiva la construcción impersonal, que es la estructura esperable para toda activa con complemento oracional; ello probaría que el acusativo está sintácticamente desligado del verbo regente y, consecuentemente, no pasaria a ser su sujeto en la pasiva. De este modo, habrian de estar documentadas frases como:

(12b) *in uincla te duci iubebitur.

Sin embargo, el tipo (12b) no aparece documentado, sino que para estos casos la construcción pasiva sigue siendo de $\mathrm{NcI}$; en ellas el nominativo es al mismo tiempo paciente del verbo principal y del infinitivo:

(14) Cic. Ph. 2,79 iussus est renuntiari consul.

(15) Liu. XXXVII 56,5 haec omnia ... regi Eumeni iussa dari.

(16) Liu. XXII 60,4 alii nullam publice impensam faciendam nec prohibendos ex priuato redimi.

Me parece que este tipo de frases es decisivo para la interpretación de las estructuras de complementación que presentan estos verbos en activa, porque confirman que el acusativo sigue funcionando, a pesar de su caracterización semántica, como complemento del verbo regente y no como sujeto del infinitivo. Como consecuencia, demuestran que el acusativo y el infinitivo no constituyen una estructura oracional en distribución con 
verbos impresivos, sino que son complementos regidos individualmente por el predicado. Al mismo tiempo, obligan a reconsiderar la validez de los criterios semánticos que se han considerado característicos del complemento nominal de los verbos impresivos en latín.

4.3. El análisis precedente nos lleva a concluir que las construcciones pasivas de $\mathrm{NcI}$ que documentan los verbos impresivos no presentan ninguna anomalia, sino que resultan de una transformación totalmente regular: el acusativo complemento de la activa pasa a nominativo sujeto en la pasiva, y el infinitivo segundo complemento nominal de la activa permanece como tal, como es lo esperable en las estructuras de complementación doble.

5. No admiten una interpretación tan clara las pasivas personales cuando se documentan con los verbos de lengua, de pensamiento, etc. $\mathrm{Si}$ para los verbos impresivos se han considerado una transformación regular de estructuras activas de Ac. + Infinitivo, en principio sería conveniente que en este segundo tipo de verbos pudiera mantenerse el mismo análisis. Para ello existen, sin embargo, algunas dificultades:

1) Frente a los verbos impresivos, los de lengua, pensamiento, etc. no pueden considerarse de doble complementación, es decir, no presentan en su estructura lugar sintáctico para dos complementos, por lo que el análisis de los complementos de infinitivo en Ac. + Infinitivo, regidos individualmente por el predicado, resulta para ellos un tanto forzado.

2) La coexistencia sincrónica de las construcciones pasivas de NcI con las impersonales de AcI obligaría a mantener un doble análisis de la complementación activa de infinitivo: Ac. + Infinitivo, transformable en NcI pasivo, en unos casos, y AcI relacionado con verbo pasivo + AcI en otros, lo cual resulta inadecuado tratándose de las mismas distribuciones.

5.1. El tratamiento que han recibido estas estructuras en la bibliografia moderna es diverso. Bolkestein ${ }^{8}$, que estudia el problema para los uerba dicendi, no aporta una solución sintáctica aceptable. Para ella, las pasivas personales de estos verbos son sintácticamente irregulares porque considera que las frases activas con complemento de persona en acusativo son agramaticales, es decir, estos verbos en la época clásica ya no pueden complementarse con un acusativo de persona: *dico te. Consecuentemente, el nominativo de la pasiva no puede obedecer a una transformación regular. Por ello explica las estructuras de $\mathrm{NcI}$ en términos semánticos y pragmáticos, pero no sintácticos.

8 «AcI and ut-Clauses with uerba dicendi in Latin», Glotta 54, 1976, p. 272. 
E. Sánchez Salor ${ }^{9}$ critica el proceder de Bolkestein, argumentando que frases como dico te, que ella considera agramaticales, están atestiguadas en latín, como puede verse en los siguientes casos:

(17) Ter. Andr. 85-86 Phaedrum aut Cliniam dicebant Niceratum.

(18) Cic. Orat. 110 cum dico me, te, Brute, dico ${ }^{10}$.

De ello concluye que la pasiva del tipo dicor responde a la misma estructura que la del tipo admoneor, considerada regular en cuanto que relacionada con admonere me. Sin embargo, el trabajo mencionado no aporta más datos que los del verbo dico, por lo que parece suponer que todos los verbos cuyas pasivas pueden adoptar la forma $\mathrm{NcI}$ han de presentar la misma estructura, lo cual no tiene por qué ser cierto. De hecho, algunos verbos de pensamiento, como existimo, que tienen pasivas de $\mathrm{NcI}$ no la comparten, pues en latín no se documenta un ${ }^{*}$ existimo $t^{11}$. Por eso, entiendo que la solución que se aporta en este trabajo no explica todos los casos ni soluciona en definitiva cómo puede mantenerse el análisis de Ac. + Infinitivo para la complementación activa de verbos de un solo complemento. Por ello me parece necesaria una descripción formal de las construcciones de $\mathrm{NcI}$ que, dando cuenta del mayor número de datos que sea posible, considere las estructuras sintácticas de los verbos con los que se documenta.

5.2. Las clases semánticas de verbos que admiten en pasiva la coexistencia de construcciones de AcI y de NcI, presentan en voz activa unas estructuras de complementación que son comunes a todas y otras que son especificas de alguna de ellas. Los verbos de lengua (dico, nuntio, pronuntio, por ejemplo) son susceptibles de aparecer con un complemento de persona en dativo además del AcI:

(19) Liu. I 22,7 nuntiate regi uestro regem Romanum deos facere testes.

La transformación de esta frase a la voz pasiva da lugar a una construcción impersonal; en otras palabras, cuando el complemento de perso-

9 "Intento de explicación de la pasiva personal en latín», EMERITA 51, 1983, p. 333.

10 No me parece acertada la objeción de que estos acusativos no sean complementos sintácticos sino propios de la función metalingüistica (S. Mariner, comunicación oral), pues, si bien el ejemplo de Cic. Orat. 110 admite esa interpretación, frases del tipo Cic. Brut. 32 quos paulo ante diximus (cf. asimismo Caes. B.G. III 26,2; Cic. Orat. 219) parecen más bien indicar que el acusativo es un complemento estructural. El mismo tipo de estructura se documenta en castellano con el verbo 'decir': "no digo esos libros, digo los que están encima de la mesa» frente a «no he dicho 'esos árboles', he dicho 'esos faroles'”.

${ }_{11}$ Cf. Lewis-Short, A Latin Dictionary, Oxford 1879 (reimpr. 1984), s.u. existimo. 
na en dativo está presente en la voz pasiva, ésta no adopta la estructura de NcI:

(20) Liu. IV 39,4 cum circumsedentibus nuntiatum esset castra deserta esse.

Parece, pues, que la presencia del dativo de persona en la versión activa es un índice de que la estructura de complementación es oracional. Este dato viene confirmado, en cierto modo, por aquellos verbos que sólo admiten en pasiva la construcción impersonal, persuadeo, por ejemplo, porque son verbos en cuya estructura siempre puede aparecer el complemento en dativo. Todo ello permite concluir que la presencia del dativo en la voz pasiva es incompatible con un sujeto personal. Este dato no explica, sin embargo, el hecho de la coexistencia de ambas construcciones cuando el complemento en dativo no aparece, pero sugiere que debe existir otra estructura de complementación que no bloquee el sujeto personal. Creo que esa estructura de complementación podría ser la de acusativo-predicativo.

5.3. La complementación de acusativo-predicativo es común a todos los verbos que pueden pasivizar en $\mathrm{NcI}$ :

(21) Hor. Sat. II 2,130 insanum te omnes pueri clamentque puellae.

(22) Cic. Com. 45 quem ego si ferrem iudicem.

(23) Liu. XLV 19,11 quia tantas praesentes eius opes cernat.

(24) Liu. IX 16,9 quos sontes comperit.

(25) Cic. Verr. III 190 eum qui hoc facit auarum possumus existimare.

(26) Liu. XXI 41,10 uelut si seruos uideatis uestros arma ... ferentes.

(27) Liu. XXIV 27,3 pronuntiant eos praetores.

La transformación a la voz pasiva de este tipo de estructuras da lugar a una construcción con sujeto personal en nominativo y un predicativo en concordancia con él. Esta construcción está ciertamente a un paso de la de $\mathrm{NcI}$ y es, me parece, con la que hay que relacionar esta última.

La complementación predicativa es el único rasgo estructural que diferencia los uerba dicendi y los del tipo persuadeo, y es al mismo tiempo la que hace que los primeros admitan las dos estructuras de pasiva y los segundos sólo las impersonales: ambas clases tienen en común la variante dativo + AcI, pero mientras que los dicendi tienen además la de Ac.-pred., persuadeo no la comparte: el dativo de persona es incompatible con la complementación predicativa, razón por la cual lo es también con el sujeto personal de la pasiva.

Los demás verbos que admiten las dos estructuras pasivas, comparten con los dicendi la complementación de AcI en alternancia con Acusativopredicativo; no presentan, sin embargo, dativo de persona. 
Véase, pues, que la complementación predicativa está en la base de todos los verbos que documentan la coexistencia entre pasivas de AcI y de $\mathrm{NcI}^{12}$.

5.4. Partiendo de estas estructuras de complementación existen dos explicaciones posibles para las construcciones de $\mathrm{NcI}$ :

1) La pasiva de NcI puede haberse producido por una contaminación de las dos estructuras de complementación activa, la predicativa y la de AcI, que presentan cierta similitud formal, si bien no estructural: la predicativa del tipo dico te bonum, a la que corresponde una pasiva con sujeto personal del tipo tu diceris bonus, y la de AcI del tipo dico te ire, cuya pasiva correspondiente sería una impersonal del tipo dicitur te ire, habrían dado lugar al tipo $\mathrm{NcI} t u$ diceris ire. Un dato que puede haber favorecido la contaminación estructural es el hecho de que el modelo predicativo constituido por dico te bonum es fácilmente interpretable como dico te bonum (esse) ${ }^{13}$; la pasiva de esta frase podría adoptar una forma o la otra según la interpretación que recibiera.

2) Las estructuras de acusativo-predicativo constituyen el modelo formal para el análisis de acusativo-infinitivo de la activa; esta última forma de complementación no sería considerada como una estructura de dos complementos nominales, acusativo + nombre verbal, sino que se analizaría como un acusativo con un elemento predicativo, expresado, en este caso, no por medio de un adjetivo, sino por un nombre verbal adjetivado, el infinitivo; cuando ese nombre verbal es de morfología invariable (formas en -re, -ri, -isse), no establece concordancia con el nombre al que determina:

(28) Liu. II 20,12 dictator ... uouisse fertur ac pronuntiare militi praemia.

Si el infinitivo adjetivado tiene marcas de género, número y caso establece con el nombre al que determina las relaciones de concordancia morfológica que el sistema del latín determina, que son las mismas que para el adjetivo predicativo, tanto en activa como en pasiva:

(29) Liu. IV 41,1 oratio incompta fuisse dicitur.

12 Más datos de esta relación pueden hallarse comparando las páginas que Kühner-Stegmann (Lateinische Grammatik, Hannover 1912 [reimpr. 1971]) dedican a los verbos de complementación predicativa (II 1, p. 291 ss.) con las de los verbos de pasiva personal (II 1, p. 705 ss.).

13 En esta misma reinterpretación se basa A. Hahn para explicar el origen de los AcI declarativos. Cf. "Genesis of the infinitive with Subject-Accusative», TAPhA 31, 1950, pp. 117-29. 
Dentro de esta explicación encajan perfectamente todos los casos en que la estructura activa presenta un complemento en acusativo y una forma de participio en concordancia con él, que no permiten decidir si se trata de complementos predicativos o de oraciones de infinitivo con la cópula elidida:

(30) Liu. XXXIX 31,17 pronuntiauitque eorum maxime opera hostes fusos.

La opción entre los dos análisis es irrelevante para los verbos regentes que estamos tratando aquí, los que pasivizan en NcI: cualquiera de los dos es igualmente posible, porque su estructura activa documenta tanto complementos predicativos como oraciones de AcI. En cambio con aquellos que no admiten la pasiva de $\mathrm{NcI}$, y consecuentemente tampoco presentan en activa estructuras de complementación predicativa, este tipo de frases han de interpretarse como de AcI con cópula elidida, pues de otro modo podrian pasivizar en $\mathrm{NcI}$ :

(31) Liu. III 21,7 tibi persuadeas me ita accepturum ut...

5.5. Para las dos interpretaciones propuestas es imprescindible partir del esquema de complementación predicativa; en el primer caso porque es el tipo de estructura sobre la que se produce la analogía o contaminación; en el segundo, porque proporciona el modelo de relación teórico que permite el nuevo análisis de los complementos con infinitivo. También se explica de este modo por qué no presentan pasivas de NcI todos los verbos que en activa rigen complementos de infinitivo: este tipo de construcción pasiva sólo puede producirse en aquellos verbos cuyo marco estructural admite una forma de complementación que la posibilita. La forma de complementación, como se ha visto, no es un simple acusativo, sino un acusativo-predicativo.

5.6. Cualquiera de las dos interpretaciones ofrece ventajas: por un lado asocia la construcción personal con estructuras no de dos complementos nominales - explicación que no se adecúa al marco de un solo complemento que presentan estos verbos- , sino con una estructura de un único complemento sintáctico, el acusativo, determinado por un predicativo, que permite que se produzca bien la contaminación estructural, bien la reinterpretación de la complementación activa. Por otro, regulariza el comportamiento de las construcciones de NcI, en cuanto que las explica como transformaciones de complementos predicativos en acusativo, que es la transformación regular de toda la pasiva latina. 
5.7. La asociación del nominativo de la pasiva con un acusativo de la activa puede parecer obvia. Sin embargo, la omisión de esta clase de asociación ha producido errores en la interpretación de la pasiva latina en algunos trabajos recientes. E. Sánchez Salor ${ }^{14}$ relaciona la frase activa

(32) Ioannes donauit libros fratri meo

con la pasiva

(32a) frater meus donatus est libris a Ioanne.

Una relación de este tipo, que supone la posibilidad de que un dativo de la versión activa pase a sujeto en nominativo de la pasiva, no es acertada porque no coincide con los datos del latín. Como es bien sabido, la pasiva (32a) se corresponde con una activa con complemento de persona en acusativo, forma de complementación que también admite el marco estructural de este verbo ${ }^{15}$ :

(32b) Ioannes donauit fratrem meum libris.

La misma interpetación de un sujeto pasivo en nominativo procedente de un dativo de la activa aparece en el artículo de $\mathrm{M}$. Lemaire ${ }^{16}$ para el verbo interdico. La autora intepreta que la frase

(33) Gell. XV 11,4 aliquis interdicitur aliqua re

procede de

(33a) interdicere alicui aliqua re,

sin tener en cuenta que el verbo interdico, como dono, posee también una estructura de complementación activa en la que el complemento de persona aparece en acusativo:

(33b) interdicere aliquem aliqua re,

atestiguado en César ${ }^{17}$.

14 "La construcción pasiva en verbos latinos de tres lugares», Estudios de Filologia Latina, II, Granada 1980, p. 177.

15 Cf. Cic. Arch. 5 itaque hunc ... ciuitate ... donarunt.

16 "Un aspect de l'échange des arguments du verbe: les verbes à double régime de l'objet en Latin», BSL 80, 1985, p. 310.

17 Lewis-Short, A Latin Dictionary, s.u. interdico. 
5.8. A manera de conclusión, puede decirse que todo sujeto personal de la voz pasiva se relaciona con un complemento activo en caso acusativo. Las construcciones de $\mathrm{NcI}$ obedecen al mismo principio, por lo que han de ponerse en relación con estructuras de complementación predicativa en acusativo. Por otro lado, el rasgo que explica la coexistencia de construcciones personales ( $\mathrm{NcI}$ ) e impersonales (AcI) de la voz pasiva no es semántico, sino sintáctico: admite ambas construcciones todo verbo cuya complementación activa presente, al menos, estas dos variantes: AcI/Acusativo predicativo.

6. Una vez descritas las condiciones sintácticas que explican la variación sintáctica de la estructura pasiva de los verbos que documentan las dos variantes, habría que explicar también qué es lo que determina la elección de una u otra en las frases latinas.

6.1. La gramática tradicional la atribuye en algunas ocasiones a particularidades del contexto. Ernout-Thomas ${ }^{18}$ señalan que es preferida la construcción impersonal cuando el verbo pasivo aparece en perfecto perifrástico, haciendo notar que, en todo caso, se trata de una preferencia, no de una elección sistemática, lo cual debilita la validez de la asociación. E. Sánchez Salor ${ }^{19}$ explica esta preferencia por el significado aspectual resultativo que tienen estos perfectos, que, según él, es incompatible con un sujeto personal. Dos aspectos de su explicación me parecen dudosos:

1) por un lado, no es seguro que pueda atribuirse de un modo sistemático un significado de aspecto resultativo al perfecto latino, cuando parece que el sistema aspectual del latín no está tan sólidamente establecido ${ }^{20}$;

2) en todo caso no se ve claro por qué el aspecto resultativo ha de ser incompatible con un sujeto personal.

Sea como sea, habida cuenta de que no pueden hallarse distribuciones fijas para una y otra construcción, no me parece que sea posible delimitar su presencia atendiendo al contexto.

6.2. La gramática tradicional ha acudido otras veces a cuestiones de énfasis para explicar la elección; la construcción impersonal hace hincapié sobre la idea declarativa, mientras que en la de NcI esta idea aparece difuminada $^{21}$. En términos parecidos aborda el problema la gramática funcional cuando explica la elección de estas construcciones en razón de la asignación de funciones pragmáticas. A.M. Bolkestein ${ }^{22}$ indica que el

\footnotetext{
18 Syntaxe, p. 327.

19 «Intento de explicación...», p. 341 ss.

20 Ernout-Thomas, Syntaxe, pp. 323-324.

21 Ernout-Thomas, p. 327.

22 «Embedded Predications, Displacement and Pseudo-argument Formation in Latin", Predication and Expression in Functional Grammar, Londres 1981, p. 96 ss.
} 
acusativo de la activa pasa a nominativo de un NcI cuando recibe la función pragmática de foco. Esta función aparece definida en la gramática funcional de S. Dik ${ }^{23}$ del modo siguiente: «el foco presenta lo que es relativamente la información más sobresaliente o importante en la localización dada». Cuando esta función recae sobre el verbo principal, éste aparece como centro de la información, adoptando la frase, por tanto, la construcción impersonal; esto viene a equivaler al realce de la idea declarativa indicado por Ernout-Thomas. Desde este punto de vista también se entiende la preferencia de la construcción impersonal cuando el verbo aparece modificado por alguna determinación adverbial; al fin y al cabo, lo que hace el adverbio es realzar el contenido del verbo, convertirlo en foco de atención, en último caso. En mi opinión, una explicación en estos términos es la que mejor da cuenta de los datos del latín, siempre y cuando vaya acompañada de una descripción previa de las condiciones sintácticas que permiten esa asignación de funciones pragmáticas; esto es lo que falta en el trabajo de Bolkestein mencionado, y sin embargo es imprescindible, porque sin la explicación sintáctica previa no hay modo de entender por qué razón no puede recibir la función de foco cualquier acusativo de un AcI, pasando así a nominativo de NcI. En cambio, este hecho queda explicado si al criterio de la función pragmática se antepone la descripción sintáctica que hemos señalado en las páginas precedentes; la sintaxis limita, en todo caso, las posibilidades de la pragmática.

7. Los puntos desarrollados a lo largo de estas páginas resuelven satisfactoriamente, en mi opinión, los problemas planteados en 3., en la medida en que explican:

1) El análisis adecuado para la complementación activa que da lugar a $\mathrm{NcI}(4.1,4.2,5.3$ y 5.4$)$.

2) Las razones de la exclusividad de la construcción del NcI en pasiva para los verbos impresivos (4.2), las de su coexistencia con pasivas de AcI con los demás verbos que las documentan $(5.3$ y 5.4$)$ y las condiciones de selección de una u otra para estos últimos (6.2).

3) La regularidad de tales construcciones en todos los casos (4.3 y 5.8).

Las conclusiones que pueden extraerse, a manera de recapitulación, de lo expuesto aqui son las siguientes:

1) Las construcciones de NcI pueden considerarse perfectamente regulares y responden a los mecanismos transformatorios que guian toda la pasiva latina.

${ }^{23}$ Functional Grammar, Amsterdam 1978 (trad. esp. Madrid 1981, p. 38). 
2) En los verbos que no admiten pasivas impersonales, $\mathrm{NcI}$ se explica como pasivización de sus estructuras de complementación activas, que no son oraciones de AcI, sino complementos dobles formados por un acusativo nominal y un nombre de acción, el infinitivo.

3) En los verbos que admiten la coexistencia de construcciones personales e impersonales de pasiva, las de $\mathrm{NcI}$ han de explicarse a partir de estructuras de complementación predicativa, bien como producto de una contaminación estructural, bien como transformaciones de estructuras reanalizadas como predicativas, con adjetivación del nombre verbal.

4) En los verbos que pueden presentar las dos versiones pasivas, aparece la de NcI cuando el acusativo de la activa recibe la función pragmática de foco. Cuando esta función recae sobre el verbo, la frase adopta la construcción impersonal.

M. ${ }^{\mathrm{a}}$ ESPERANZA TORREGO 